\title{
A parametric study on inelastic buckling in steel cylindrical shells with circular cutouts
}

\author{
S. Miladi • M. S. Razzaghi
}

Received: 30 August 2013/ Accepted: 27 January 2014/Published online: 19 March 2014

(C) The Author(s) 2014. This article is published with open access at Springerlink.com

\begin{abstract}
Thin-walled cylindrical shells are important components of many industrial complexes. Most of these components have circular cutouts in manholes and pipe-toshell junctions. Performance of cylindrical shells due to the extreme loading conditions shows that buckling is the major failure mode in such components. This study aims to indicate the effect of circular cutouts on buckling capacity of cylindrical shells due to pure axial compression. To this end, cylindrical shells of different geometric specifications and various arrangements and sizes of cutouts were considered. Numerical nonlinear analyses were conducted using ANSYS software. Result of this study revealed that cutouts can play a noticeable role in creating stress concentration and affect destructively the stability of structures. It is shown that there is a noticeable difference between the effects on cutouts in buckling of thinner shells and thicker ones. Cutouts reduce the local buckling capacity of shell about $10-15 \%$ in the cylindrical shells, with the diameter to thickness ratio of less than 1,000. Meanwhile in shells with diameter to thickness, more than 1,000 such cutouts reduce the shell capacity about 30-35\%.
\end{abstract}

Keywords Cylindrical shells · Buckling · Circular cutout

S. Miladi · M. S. Razzaghi $(\bowtie)$

Department of Civil Engineering, Qazvin Branch, Islamic Azad

University, QIAU, Nokhbegan Blvd, Qazvin, Iran

e-mail: razzaghi.m@gmail.com

S. Miladi

e-mail: sina_miladi@yahoo.com

\section{Introduction}

Shells have several applications in engineering structures and most particularly in civil engineering, and mechanical engineering, architecture, aerospace and marine industries.

The shell buckling is the most important failure mode in most of the thin-walled structures. The buckling of thinwalled cylindrical shells is much more important during earthquakes. The shell buckling depends on many parameters, one of which is cutout. The cutouts are formed for access openings, pipe line passage and so on and can affect the stability of these structures. Therefore, in designing the tanks and marine structures, it is essential to discover how cutouts affect the loading capacity and buckling behavior of cylindrical shells. In this regard, wide investigations have been conducted by the researches.

During the past decades, several researches have been conducted on the linear elastic buckling of shells. However, the empirical investigations done by Arbocz and Hol (1991) and Jullien and Limam (1998) proved that the buckling capacity values of thin-walled cylindrical shells are lower than those predicted through Timoshenko's classical theory (Timoshenko and Gere 1961). Toda (1983) has empirically studied the cylindrical shells with circular cutouts under axial compression; he investigated the stiffness effect around the cutouts as well. Jullien and Limam (1998) studied the effects of circular, square and rectangular cutouts on the buckling of cylindrical shells under axial compression using CASTEM2000, a finite element software, and presented a parametric relation based on the shapes and dimensions of the cutouts. Van Dyke (1965) computed the stress distribution around an opening in the cylindrical shells with cutout under axial, torsional and internal compressive loadings. Tennyson (1968) has empirically studied the effects of circular cutout of 
cylindrical shells under axial compression and compared the obtained buckling load values with the analytical results of Van Dyke (1965). Yeh et al. (1999) have assessed, analytically and empirically, the torsional buckling of cylindrical shells with cutout. Brogan and Almorth (1970) have studied the effects of rectangular cutout on the buckling load of cylindrical shells and compared the obtained experimental results with those of STAGS program. Starnes (1970) has empirically studied the effect of circular cutout on the buckling of cylindrical shells under axial compression and obtained a high limit for buckling load by Reyleigh-Ritz method. Han et al. (2006) has applied nonlinear numerical method using ANSYS software to study the size and location effects of square cutouts on the thin- and thick-walled cylindrical shells and presented several parametric relations based on his analytical and empirical results. Tafreshi (2002) has applied ABAQUS software to investigate the buckling and post-buckling behaviors of composite cylindrical shells with rectangular cutout under axial and internal compressive loadings. He has concluded that the buckling capacity load increases with increasing the internal compression. Shariati and Mahdizadeh Rokhi (2010) have studied the effects of elliptical cutouts with different sizes and angles on the buckling of cylindrical shells under axial compressive load. Hilburger et al. (1998) has assessed the buckling of composite cylindrical shells with square cutouts under axial and internal compressive loadings.

This study aims to investigate the effect of circular cutouts on inelastic buckling and post-buckling behavior of cylindrical shells. In this study, the effects of different arrangements of the circular cutouts on buckling and postbuckling behavior of the cylindrical shells are investigated. To this end, FEM models were prepared in ANSYS (2009) environment, and nonlinear analyses have been performed. Herein, the effects of various arrangements of cutouts on buckling of cylindrical shells of different geometric specifications were studied.

\section{Modeling}

In this research, the steel cylindrical shells with circular cutout, summarized in Table 1, have been modeled and analyzed using ANSYS 12.1 software to study the effect of cutout on the loading capacity and buckling behavior of cylindrical shells. As indicated in Fig. 1, D and $\mathrm{H}$ denote diameter and height of the cylinder, respectively. The parameter $t$ is the thickness of the shell and $h$ denotes distance of the center of the cutout to the bottom of the shell. The analyses have been conducted by subjecting the structure to the pure axial load and lateral loading. As shown in Table 1, the height of the all cylinders was
Table 1 Geometric specifications of cylindrical shells

\begin{tabular}{llllllll}
\hline$n$ & $D(\mathrm{~m})$ & $t(\mathrm{~m})$ & $H(\mathrm{~m})$ & $D / t$ & $H / D$ & $d(\mathrm{~cm})$ & $h(\mathrm{~cm})$ \\
\hline 1 & 5 & 0.010 & 14.75 & 500 & 2.95 & $30-50-60-80-100$ & $d-2 d-4 d$ \\
2 & 5 & 0.012 & 14.75 & 417 & 2.95 & $30-50-60-80-100$ & $d-2 d-4 d$ \\
3 & 5 & 0.020 & 14.75 & 250 & 2.95 & $30-50-60-80-100$ & $d-2 d-4 d$ \\
4 & 10 & 0.010 & 14.75 & 1,000 & 1.48 & $30-50-60-80-100$ & $d-2 d-4 d$ \\
5 & 10 & 0.012 & 14.75 & 833 & 1.48 & $30-50-60-80-100$ & $d-2 d-4 d$ \\
6 & 10 & 0.020 & 14.75 & 500 & 1.48 & $30-50-60-80-100$ & $d-2 d-4 d$ \\
7 & 15 & 0.010 & 14.75 & 1,500 & 0.98 & $30-50-60-80-100$ & $d-2 d-4 d$ \\
8 & 15 & 0.012 & 14.75 & 1,250 & 0.98 & $30-50-60-80-100$ & $d-2 d-4 d$ \\
9 & 15 & 0.015 & 14.75 & 1,000 & 0.98 & $30-50-60-80-100$ & $d-2 d-4 d$ \\
10 & 20 & 0.010 & 14.75 & 2,000 & 0.74 & $30-50-60-80-100$ & $d-2 d-4 d$ \\
11 & 20 & 0.012 & 14.75 & 1,667 & 0.74 & $30-50-60-80-100$ & $d-2 d-4 d$ \\
12 & 20 & 0.015 & 14.75 & 1,333 & 0.74 & $30-50-60-80-100$ & $d-2 d-4 d$ \\
\hline
\end{tabular}

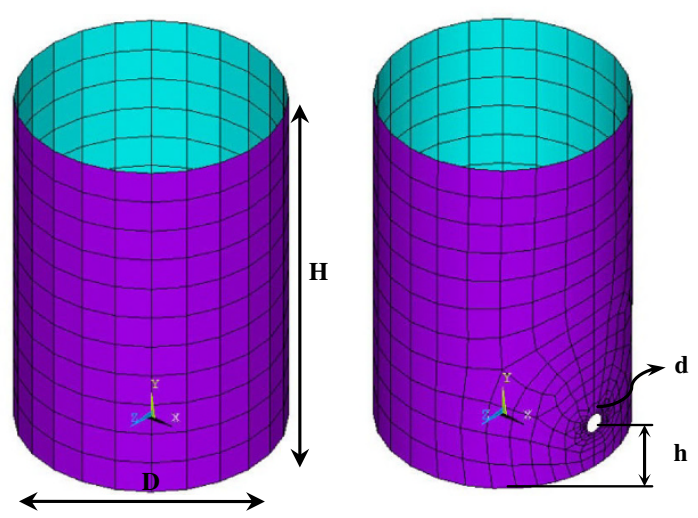

Fig. 1 Geometry and model of finite element of steel cylindrical shells with and without cutout

considered to be about $14.75 \mathrm{~m}$. This height belongs to medium cylinders in which changing the height does not make noticeable change in buckling due to pure axial loads. All of the shells were fixed at the bottom and completely free at the top.

Herein, the nonlinear static analysis has been used. The behavior of material is considered bi-linear and its specifications are, steel ST37 with elasticity of $E=2.1 e 11 \frac{\mathrm{N}}{\mathrm{m}^{2}}$ and yielding stress of $F_{\mathrm{y}}=240 \mathrm{MPa}$. The curve slope of stress-strain behavior of steel after yield point is $2 \%$ of its linear elasticity module. The heights of all samples are constant and the shell has been fixed at the bottom, concerning the boundary condition. The shell is considered to have large displacement and the Von Mises failure model is considered with kinematic hardening.

The 8 node shell 281 elements have been used in modeling (Fig. 2). These elements have the capability of considering large displacement and nonlinear behavior of the structural material and therefore are appropriate for solving the problems related to buckling. The elements 
have six degrees of freedom in each node including three transitional freedom and three rotational degrees of freedom.

\section{Analytical results}

The effect of cutouts on shell buckling

Cutouts can play an important role in the formation of stress concentration and affect destructively the stability of cylindrical shells. In this research, the effect of circular cutouts has been studied on the buckling of cylindrical shells. For this purpose, a circular cutout of $d=30 \mathrm{~cm}$ has been formed and modeled at the distance of $h=2 d$ from the shells' bottom; the shells' specifications have been summarized in Table 2. Then, the models have been subjected to the uniform pure axial loading which has been subjected to the free end of the cylinders. After analysis, the axial load-shortening curves are obtained for each node. The critical buckling load of the shell is obtained by

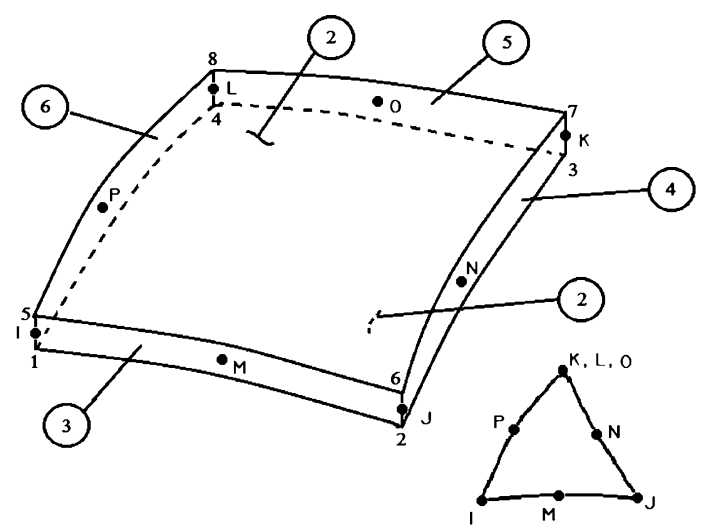

Fig. 2 Geometry of elements: 8node281 (ANSYS 2009) summation of the maximum buckling loads of supporting nodes.

By cutout formation in the shell, its buckling capacity is reduced compared to the status without cutout (see Fig. 3). This capacity loss increases $30-35 \%$ if the diameter and thickness of the shell increase (Fig. 4). Moreover, if the shell's diameter is assumed constant, the shell's buckling capacity increases with increasing its thickness (Fig. 3).

The buckling curves of cylindrical shells, with and without cutout, have been presented in Fig. 5 as per buckling-displacement. According to the figure, in case of cutout formation in the shell, the curve slope does not change in the elastic and post-buckling zones. Moreover, in the shell with cutout, the area under force-displacement curve which indicates the structure's energy dissipation shows no significant change in comparison with that of complete shell.

According to Fig. 6, with increasing the shell's thickness, no significant change is seen in the curve slope in the elastic zone, while it slightly changes in the postbuckling zone. The shell's buckling capacity as well as shell's energy dissipation increases with increasing the shell's thickness. The deformation of cylindrical shells under axial compression has been presented in Fig. 7 as an example.

The effect of cutout diameter on shell buckling

Here, a circular cutout with the diameters of $d=30,50$, 60, 80 and $100 \mathrm{~cm}$ has been formed and modeled at the distance of $h=2 d$ from the shells' floor, according to the Tables 3, 4, 5, 6 to investigate the effect of diameter change on the cylindrical shells buckling.

As indicated in Fig. 8, in the cylindrical shells with cutout, the local buckling capacity is significantly reduced;
Table 2 Buckling force and geometric specifications of selected shells (the effect of cutout formation)

\begin{tabular}{llllllllllc}
\hline$n$ & $D(\mathrm{~m})$ & $t(\mathrm{~m})$ & $H(\mathrm{~m})$ & \multicolumn{1}{c}{$D / t$} & $H / D$ & $d(\mathrm{~cm})$ & $h(\mathrm{~cm})$ & $H / h$ & $h / d$ & Pcr $\left(10^{6} \mathrm{~N}\right)$ \\
\hline 1 & 5 & 0.010 & 14.75 & 500 & 2.95 & 30 & 60 & 24.58 & 2 & 35.03 \\
2 & 5 & 0.012 & 14.75 & 417 & 2.95 & 30 & 60 & 24.58 & 2 & 44.67 \\
3 & 5 & 0.020 & 14.75 & 250 & 2.95 & 30 & 60 & 24.58 & 2 & 81.56 \\
4 & 10 & 0.010 & 14.75 & 1,000 & 1.48 & 30 & 60 & 24.58 & 2 & 61.85 \\
5 & 10 & 0.012 & 14.75 & 833 & 1.48 & 30 & 60 & 24.58 & 2 & 76.67 \\
6 & 10 & 0.020 & 14.75 & 500 & 1.48 & 30 & 60 & 24.58 & 2 & 155.54 \\
7 & 15 & 0.010 & 14.75 & 1,500 & 0.98 & 30 & 60 & 24.58 & 2 & 63.93 \\
8 & 15 & 0.012 & 14.75 & 1,250 & 0.98 & 30 & 60 & 24.58 & 2 & 76.71 \\
9 & 15 & 0.015 & 14.75 & 1,000 & 0.98 & 30 & 60 & 24.58 & 2 & 95.97 \\
10 & 20 & 0.010 & 14.75 & 2,000 & 0.74 & 30 & 60 & 24.58 & 2 & 80.15 \\
11 & 20 & 0.012 & 14.75 & 1,667 & 0.74 & 30 & 60 & 24.58 & 2 & 102.22 \\
12 & 20 & 0.015 & 14.75 & 1,333 & 0.74 & 30 & 60 & 24.58 & 2 & 127.87 \\
\hline
\end{tabular}


Fig. 3 Comparing the buckling capacity of shells with and without cutouts $(d=30 \mathrm{~cm})$

Fig. 4 The effect of shell diameter on reduction of Pcr

Fig. 5 Axial force-

displacement behavior of shells:

a $D=10 \mathrm{~m}, t=12 \mathrm{~mm}$;

b $D=15 \mathrm{~m}, t=12 \mathrm{~mm}$
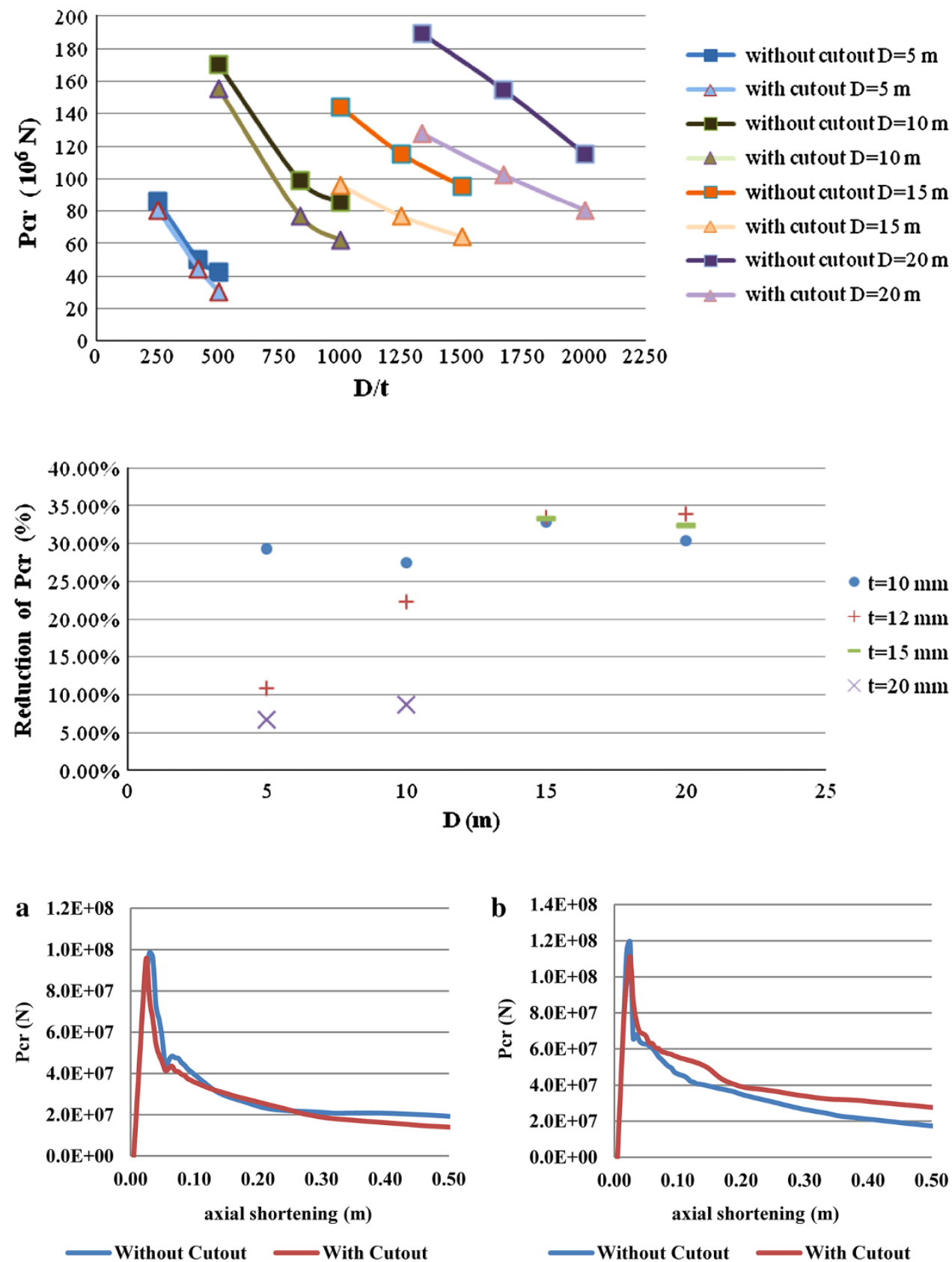

this phenomenon is more obvious in the shells with higher diameters. The buckling load keeps its reducing process with very slight slope. It can actually be said that the changing of cutout diameter in $d=30$ to $d=100$ has no significant effect on the local buckling capacity of the shells.

The shells of 5, 10, 15 and 20 meter diameters, summarized in Tables 3, 4, 5, 6, have been considered and compared to study the effect level of cutout diameter change on the buckling. In this regard, the ratio of local buckling capacity of cylindrical shells with circular cutout $\left(P_{\text {Cutout }}\right)$ of $d=30,50,60,80$ and $100 \mathrm{~cm}$ and $h=2 d$ from shell floor) to the cylindrical shells without cutout $\left(P_{0}\right)$ has been plotted as per diameter to thickness ratio of the shell (Fig. 9). According to the figure, the $P_{\text {Cutout }} / P_{0}$ ratio is about $0.85-0.95$ in the shells of 5 and $10 \mathrm{~m}$ diameters with the diameter to thickness ratio of less than 1,000 . This value is $0.65-0.75$ in the shells of 15 and $20 \mathrm{~m}$ diameters with the diameter to thickness ratio more than 1,000 .

This means that the cutout formation of $d=30 \mathrm{~cm}$ in the cylindrical shells with 5 and $10 \mathrm{~m}$ diameters will decrease buckling capacity about 10-20\%. This reduction 


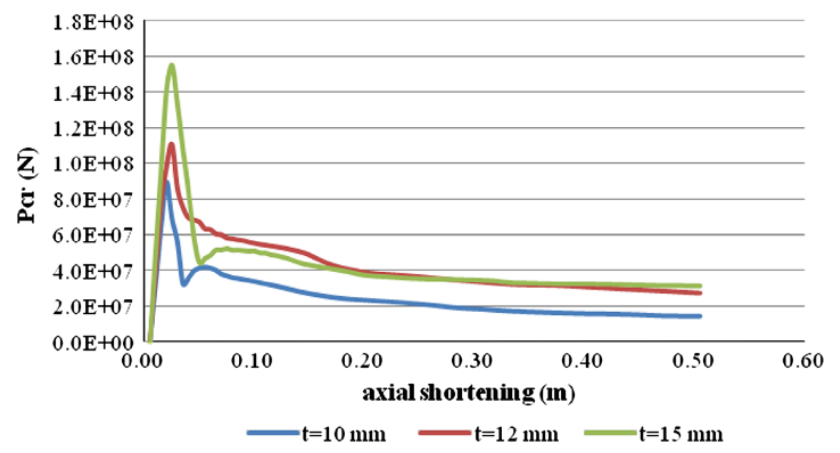

Fig. 6 Axial force-displacement behavior of cylindrical shell with cutout (the $15 \mathrm{~m}$ shell diameter is constant
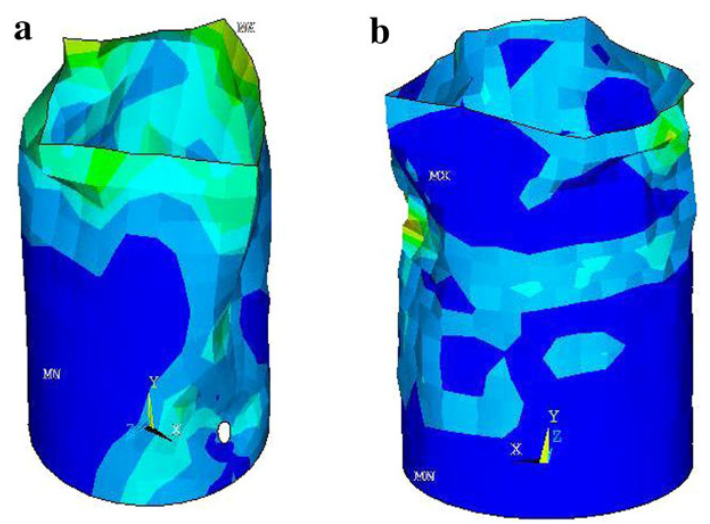

Fig. 7 The deformation of cylindrical shell under axial compression: a with cutout, $\mathbf{b}$ without cutout

will go up to $15 \%$ with increasing the cutout up to $d=100 \mathrm{~cm}$ (Fig. 10). Moreover, in the cylindrical shells with 15 and $20 \mathrm{~m}$ diameters, the cutout formation will decrease buckling capacity about $30 \%$. This reduction will go up to $35 \%$ with increasing the cutout up to $d=100 \mathrm{~cm}$.

The cylindrical shell buckling $(20 \mathrm{~m}$ diameter and $15 \mathrm{~mm}$ thickness) with the cutout of $d=30,50,60,80$ and $100 \mathrm{~cm}$ has been plotted as per buckling force-displacement (Fig. 11). According to the figure, the curve slope does not change in the elastic and post-buckling zone in case of changing the cutout diameter in the shell.
Moreover, the area under force-displacement curve which shows the structure's energy dissipation rate does not change with change in cutout diameter. Figure 12 indicates the deformed shape of the shell at the last stage of loading.

The effect of cutout location on shell buckling

A circular cutout of 50 and $100 \mathrm{~cm}$ diameters has been modeled with the distance of $h=d, 2 d$ and $4 d$ from the shell bottom to study the effect of changing in the circular cutout height from the shell bottom on the cylindrical shell buckling. The obtained analytical results have been summarized in Table 7.

The local buckling capacity of cylindrical shell with circular cutout has been plotted as per $h / d$ and presented in Fig. 13.

According to these figures, no significant change is seen in the shell buckling capacity with changing the height location of cutout. It means that the results mentioned in Fig. 10 are confirmed here and changing in the cutout location at the distances of $h=d, 2 d$ and $4 d$ from the shell bottom will cause no significant change in the buckling load reduction (see Fig. 14).

The cylindrical shell buckling $(20 \mathrm{~m}$ diameter and $15 \mathrm{~mm}$ thickness) has been plotted as per buckling forcedisplacement and shown in Fig. 15. According to this figure, no significant change is seen in the curve slope in the elastic and post-buckling zones in case of changing the cutout height from the shell bottom.

Moreover, no significant change is seen in the area under force-displacement curve, which shows the structure's ductility, with changing the cutout height position from the shell bottom. Figure 16 shows the deformation of cylindrical shell under axial compression in case of changing the cutout position in height from the bottom.

The effect of the arrangement of cutouts on shell buckling

The steel cylindrical shells with the diameters of 5 and $10 \mathrm{~m}$ and the diameter to thickness ratio of 250, 500 and 1,000
Table 3 Geometric specifications of the cutouts formed in the cylindrical shells with 5-m diameter

\begin{tabular}{llllllllrl}
\hline$n$ & $D(\mathrm{~m})$ & $t(\mathrm{~m})$ & $H(\mathrm{~m})$ & $D / t$ & $H / D$ & $d(\mathrm{~cm})$ & $h(\mathrm{~cm})$ & $H / h$ & $\begin{array}{l}h / \\
d\end{array}$ \\
\hline 1 & 5 & 0.02 & 14.75 & 250 & 2.95 & 30 & 60 & 24.58 & 2 \\
2 & 5 & 0.02 & 14.75 & 250 & 2.95 & 50 & 100 & 14.75 & 2 \\
3 & 5 & 0.02 & 14.75 & 250 & 2.95 & 60 & 120 & 12.29 & 2 \\
4 & 5 & 0.02 & 14.75 & 250 & 2.95 & 80 & 160 & 9.22 & 2 \\
5 & 5 & 0.02 & 14.75 & 250 & 2.95 & 100 & 200 & 7.38 & 2 \\
\hline
\end{tabular}


Table 4 Geometric specifications of the cutouts formed in the cylindrical shells with 10-m diameter

\begin{tabular}{rlllllllrr}
\hline$n$ & $D(\mathrm{~m})$ & $t(\mathrm{~m})$ & $H(\mathrm{~m})$ & $D / t$ & $H / D$ & $d(\mathrm{~cm})$ & $h(\mathrm{~cm})$ & $H / h$ & $h / d$ \\
\hline 1 & 10 & $0.010-0.012-0.020$ & 14.75 & $1,000-833-500$ & 1.48 & 30 & 60 & 24.58 & 2 \\
2 & 10 & $0.010-0.012-0.020$ & 14.75 & $1,000-833-500$ & 1.48 & 50 & 100 & 14.75 & 2 \\
3 & 10 & $0.010-0.012-0.020$ & 14.75 & $1,000-833-500$ & 1.48 & 60 & 120 & 12.29 & 2 \\
4 & 10 & $0.010-0.012-0.020$ & 14.75 & $1,000-833-500$ & 1.48 & 80 & 160 & 9.22 & 2 \\
5 & 10 & $0.010-0.012-0.020$ & 14.75 & $1,000-833-500$ & 1.48 & 100 & 200 & 7.38 & 2 \\
\hline
\end{tabular}

Table 5 Geometric specifications of the cutouts formed in the cylindrical shells with $15 \mathrm{~m}$ diameter

\begin{tabular}{rllllllrrr}
\hline$n$ & $D(\mathrm{~m})$ & $t(\mathrm{~m})$ & $H(\mathrm{~m})$ & $D / t$ & $H / D$ & $d(\mathrm{~cm})$ & $h(\mathrm{~cm})$ & $H / h$ & $h / d$ \\
\hline 1 & 15 & $0.01-0.012-0.015$ & 14.75 & $1,500-1,250-1,000$ & 0.98 & 30 & 60 & 24.58 & 2 \\
2 & 15 & $0.01-0.012-0.015$ & 14.75 & $1,500-1,250-1,000$ & 0.98 & 50 & 100 & 14.75 & 2 \\
3 & 15 & $0.01-0.012-0.015$ & 14.75 & $1,500-1,250-1,000$ & 0.98 & 60 & 120 & 12.29 & 2 \\
4 & 15 & $0.01-0.012-0.015$ & 14.75 & $1,500-1,250-1,000$ & 0.98 & 80 & 160 & 9.22 & 2 \\
5 & 15 & $0.01-0.012-0.015$ & 14.75 & $1,500-1,250-1,000$ & 0.98 & 100 & 200 & 7.38 & 2 \\
\hline
\end{tabular}

Table 6 Geometric specifications of the cutouts formed in the cylindrical shells with 20-m diameter

\begin{tabular}{rllllllrrr}
\hline$n$ & $D(\mathrm{~m})$ & $t(\mathrm{~m})$ & $H(\mathrm{~m})$ & $D / t$ & $H / D$ & $d(\mathrm{~cm})$ & $h(\mathrm{~cm})$ & $H / h$ & $h / d$ \\
\hline 1 & 20 & $0.01-0.012-0.015$ & 14.75 & $2,000-1,667-1,333$ & 0.74 & 30 & 60 & 24.58 & 2 \\
2 & 20 & $0.01-0.012-0.015$ & 14.75 & $2,000-1,667-1,333$ & 0.74 & 50 & 100 & 14.75 & 2 \\
3 & 20 & $0.01-0.012-0.015$ & 14.75 & $2,000-1,667-1,333$ & 0.74 & 60 & 120 & 12.29 & 2 \\
4 & 20 & $0.01-0.012-0.015$ & 14.75 & $2,000-1,667-1,333$ & 0.74 & 80 & 160 & 9.22 & 2 \\
5 & 20 & $0.01-0.012-0.015$ & 14.75 & $2,000-1,667-1,333$ & 0.74 & 100 & 200 & 7.38 & 2 \\
\hline
\end{tabular}

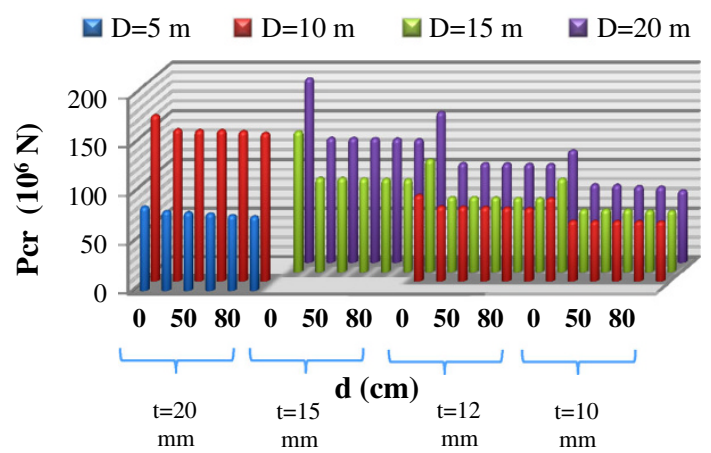

Fig. 8 Buckling force of cylindrical shell with and without cutouts as per cutout diameter

were modeled and analyzed to study the effect of increasing the cutout number on the capacity and behavior of cylindrical shell buckling. The number, arrangement and location of their circular cutouts are summarized in Table 8 . They have been subjected to the axial compression and the effect of increase in cutout number has been studied on the circumference and height of the shells. Figure 17 shows a sample of finite element model of steel cylindrical shells with the cutouts with increasing width (horizontal cutout) and height (vertical cutout).

According to Fig. 18, the buckling capacity of shell is reduced in case of forming circular cutout in the cylindrical shells of $5 \mathrm{~m}$ diameter and the diameter to thickness ratio of 250 and 500. With increasing the cutout number in the width, the buckling load keeps reducing and this fact is more obvious in the cutout of higher diameter. However, increasing cutout number in the height has no significant effect on the buckling load reduction.

Accordingly, the shell buckling capacity is reduced about $20 \%$ in the formation of cutout with $5 \mathrm{~m}$ diameter and diameter to thickness ratio of 500. This reduction is about 30 and $45 \%$ in case of increasing the cutout number in the width with 50 and $80 \mathrm{~cm}$ cutouts, respectively. If the cutout is formed in the shell of $5 \mathrm{~m}$ diameter with diameter to thickness ratio of 250 , the buckling capacity reduction is lower as the shell is thicker com- 
Fig. 9 Reduction percentage of buckling force in shells with diameters

Fig. 10 The effect of cutout diameter on local buckling capacity of cylindrical shells (shell with the diameters of 5 , 10,15 and $20 \mathrm{~m}$ )

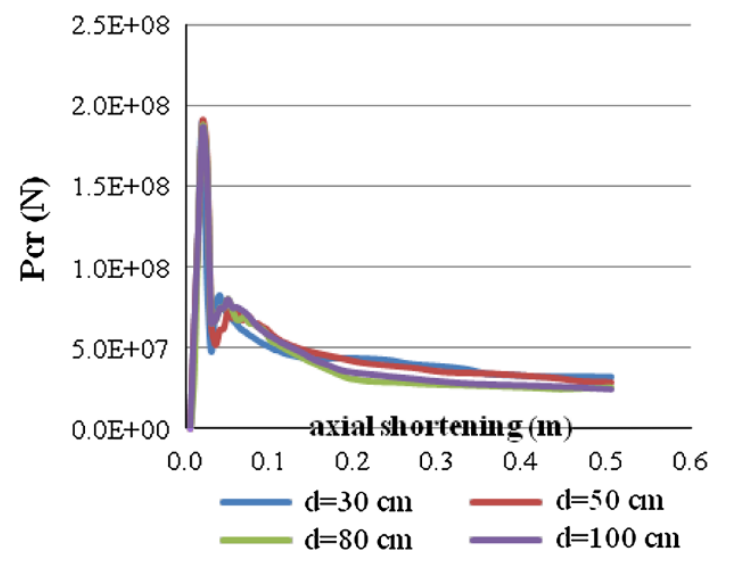

Fig. 11 Axial force-displacement behavior of cylindrical shell with cutout (shell diameter is $20 \mathrm{~m}$ and its thickness is $15 \mathrm{~mm}$ )
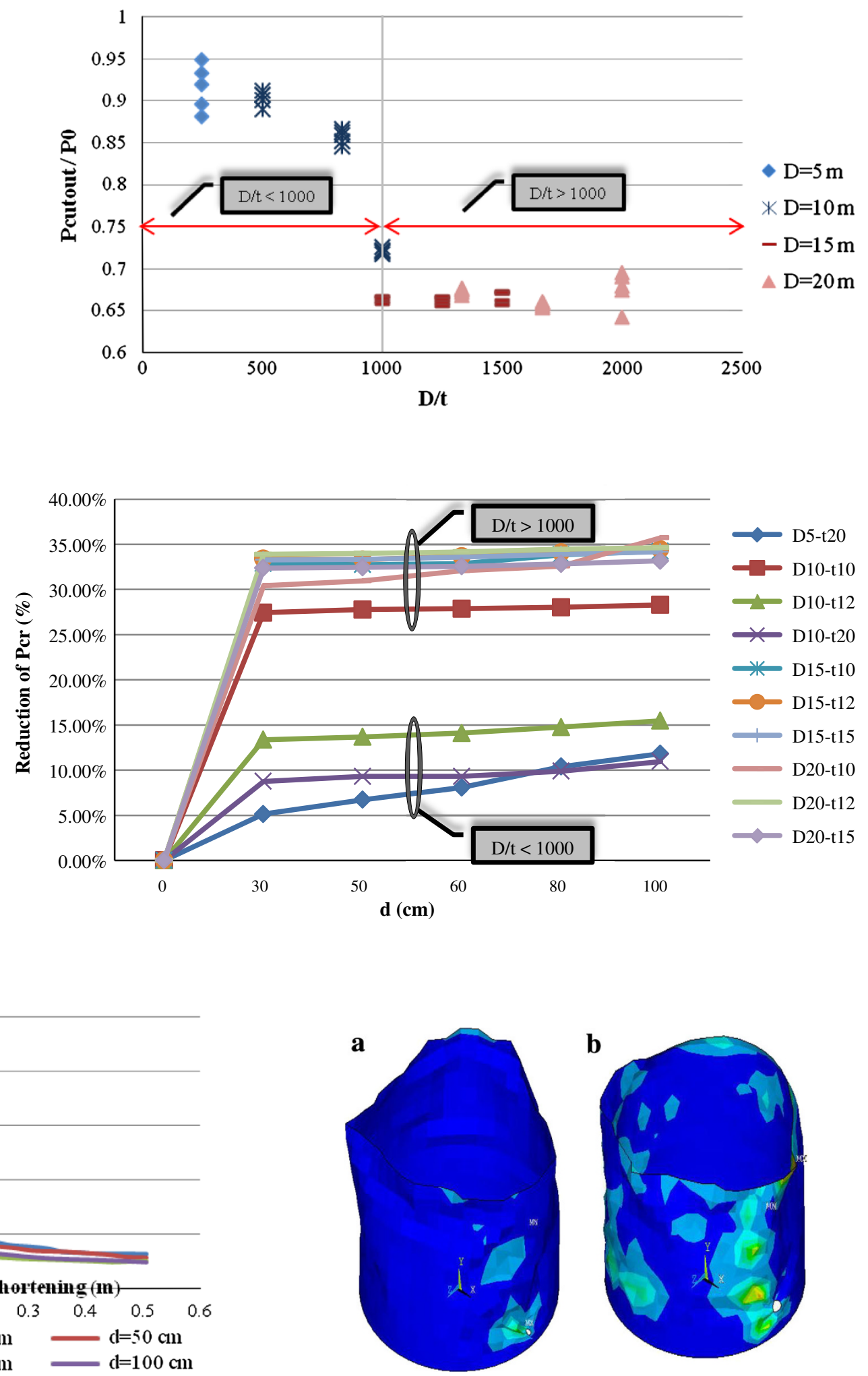

Fig. 12 The deformation of cylindrical shell with circular cutout: a $d=100 \mathrm{~cm}, D=20 \mathrm{~m} ; \mathbf{b} d=50 \mathrm{~cm}, D=20 \mathrm{~m}$ 
Table 7 Buckling force and geometric specifications of shells and cutouts of $d=50$, $100 \mathrm{~cm}$ in $d, 2 d$ and $4 d$ heights

\begin{tabular}{llllllllllll}
\hline$D(\mathrm{~m})$ & $t(\mathrm{~m})$ & $H(\mathrm{~m})$ & $D / t$ & $d(\mathrm{~cm})$ & $h(\mathrm{~cm})$ & $h / d$ & $\begin{array}{l}\mathrm{Pcr} \\
\left(10^{6} \mathrm{~N}\right)\end{array}$ & $d(\mathrm{~cm})$ & $h(\mathrm{~cm})$ & $h / d$ & $\begin{array}{l}\mathrm{Pcr} \\
\left(10^{6} \mathrm{~N}\right)\end{array}$
\end{tabular}

D10- $t 12$

\begin{tabular}{|c|c|c|c|c|c|c|c|c|c|c|c|}
\hline 10 & 0.012 & 14.75 & 833 & 50 & 50 & 1 & 76.07 & 100 & 100 & 1 & 74.65 \\
\hline 10 & 0.012 & 14.75 & 833 & 50 & 100 & 2 & 76.39 & 100 & 200 & 2 & 74.82 \\
\hline 10 & 0.012 & 14.75 & 833 & 50 & 400 & 4 & - & 100 & 400 & 4 & 74.40 \\
\hline \multicolumn{12}{|c|}{$D 15-t 10$} \\
\hline 15 & 0.01 & 14.75 & 1,500 & 50 & 50 & 1 & 63.07 & 100 & 100 & 1 & 63.09 \\
\hline 15 & 0.01 & 14.75 & 1,500 & 50 & 100 & 2 & 64.07 & 100 & 200 & 2 & 62.64 \\
\hline 15 & 0.01 & 14.75 & 1,500 & 50 & 400 & 4 & - & 100 & 400 & 4 & 62.80 \\
\hline \multicolumn{12}{|c|}{$D 15-t 12$} \\
\hline 15 & 0.012 & 14.75 & 1,250 & 50 & 50 & 1 & 76.63 & 100 & 100 & 1 & 75.83 \\
\hline 15 & 0.012 & 14.75 & 1,250 & 50 & 100 & 2 & 76.83 & 100 & 200 & 2 & 75.52 \\
\hline 15 & 0.012 & 14.75 & 1,250 & 50 & 400 & 4 & - & 100 & 400 & 4 & 75.03 \\
\hline \multicolumn{12}{|c|}{$D 15-t 15$} \\
\hline 15 & 0.015 & 14.75 & 1,000 & 50 & 50 & 1 & 95.91 & 100 & 100 & 1 & 95.02 \\
\hline 15 & 0.015 & 14.75 & 1,000 & 50 & 100 & 2 & 95.90 & 100 & 200 & 2 & 94.69 \\
\hline 15 & 0.015 & 14.75 & 1,000 & 50 & 400 & 4 & - & 100 & 400 & 4 & 94.44 \\
\hline \multicolumn{12}{|c|}{$D 20-t 10$} \\
\hline 20 & 0.01 & 14.75 & 2,000 & 50 & 50 & 1 & 79.14 & 100 & 100 & 1 & 84.29 \\
\hline 20 & 0.01 & 14.75 & 2,000 & 50 & 100 & 2 & 79.54 & 100 & 200 & 2 & 84.05 \\
\hline 20 & 0.01 & 14.75 & 2,000 & 50 & 400 & 4 & - & 100 & 400 & 4 & 84.86 \\
\hline \multicolumn{12}{|c|}{$D 20-t 12$} \\
\hline 20 & 0.012 & 14.75 & 1,667 & 50 & 50 & 1 & 101.03 & 100 & 100 & 1 & 100.66 \\
\hline 20 & 0.012 & 14.75 & 1,667 & 50 & 100 & 2 & 102.05 & 100 & 200 & 2 & 101.07 \\
\hline 20 & 0.012 & 14.75 & 1,667 & 50 & 400 & 4 & - & 100 & 400 & 4 & 100.63 \\
\hline \multicolumn{12}{|c|}{$D 20-t 15$} \\
\hline 20 & 0.015 & 14.75 & 1,333 & 50 & 50 & 1 & 127.76 & 100 & 100 & 1 & 126.65 \\
\hline 20 & 0.015 & 14.75 & 1,333 & 50 & 100 & 2 & 127.73 & 100 & 200 & 2 & 126.36 \\
\hline 20 & 0.015 & 14.75 & 1,333 & 50 & 400 & 4 & - & 100 & 400 & 4 & 125.02 \\
\hline
\end{tabular}

Cutout $-\mathrm{d}=50 \mathrm{~cm}$

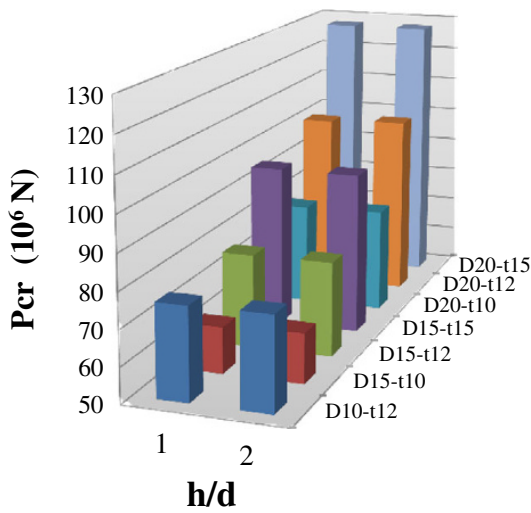

Cutout $-\mathrm{d}=100 \mathrm{~cm}$

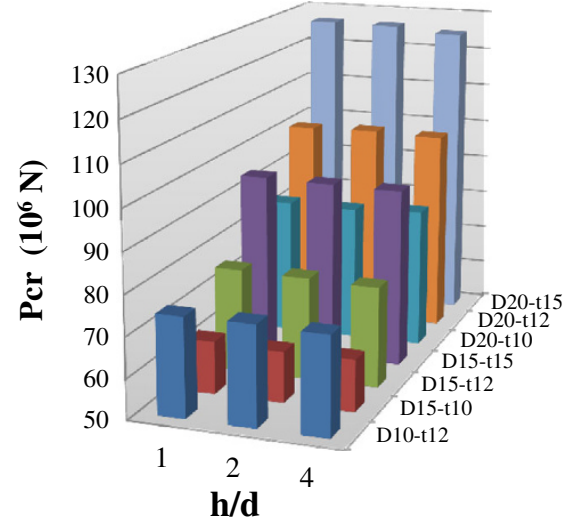

D10-t12

D15-t10

D15-t12

D15-t15

D20-t 10

D20-t12

D20-t15
D20-t15

Fig. 13 The effect of the aspect ratio of the shell on Pcr (cutout diameter $d=50 \mathrm{~cm}, d=100 \mathrm{~cm}$ ) 
Fig. 14 The effect of the location of cutouts on buckling capacity of the cylindrical shells $(d=100 \mathrm{~cm})$

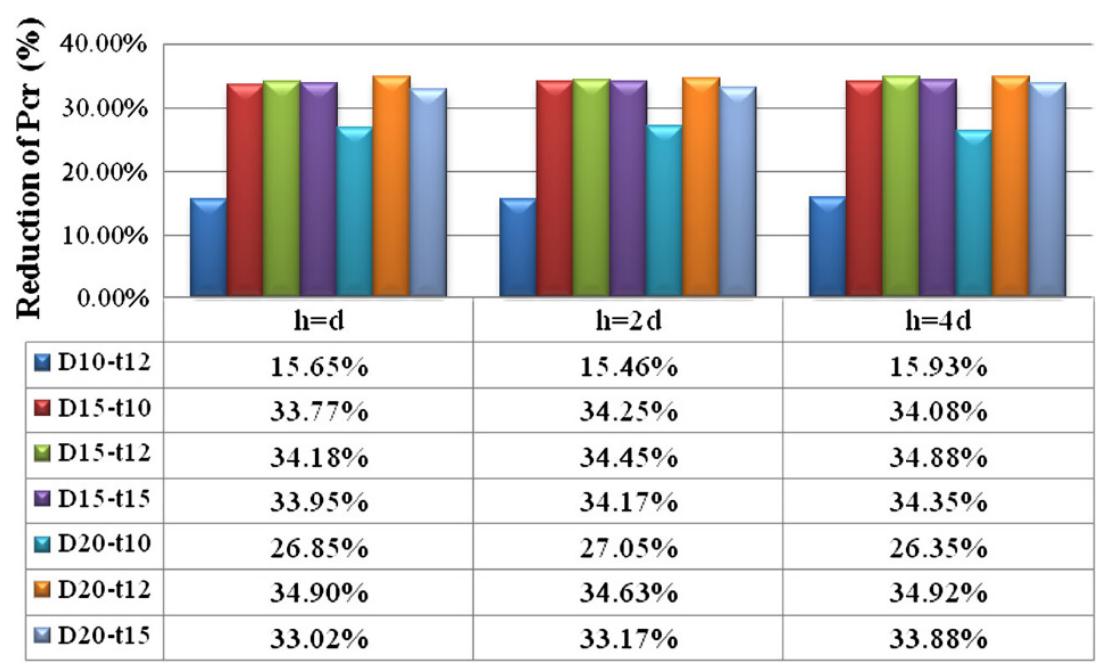

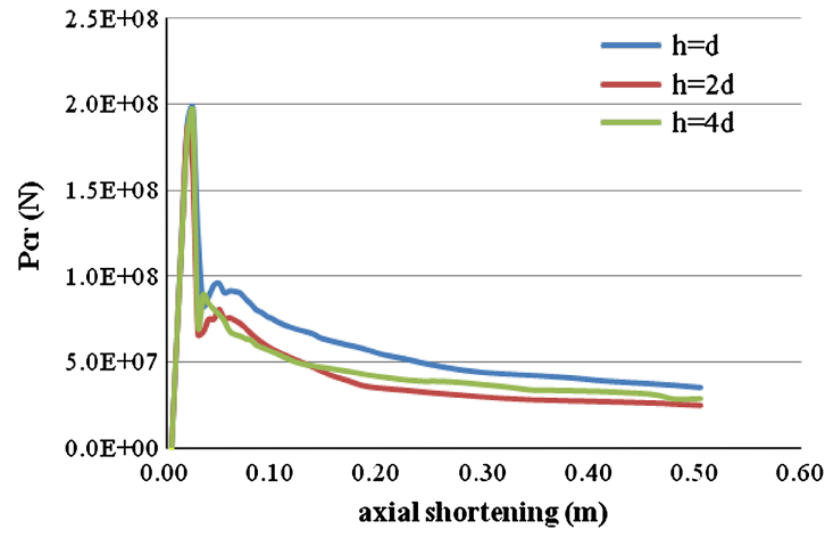

Fig. 15 Axial force-displacement behavior of cylindrical shell with cutout (shell diameters $20 \mathrm{~m}$, shell thickness $15 \mathrm{~mm}$ )

pared to previous status. The shell buckling capacity is reduced about $20 \%$ in the presence of cutout with $80 \mathrm{~cm}$ diameter which is almost half of the previous status (Fig. 19).

According to Fig. 20, the buckling capacity is reduced in case of circular cutout formation in the cylindrical shells with $10 \mathrm{~m}$ diameter and diameter to thickness ratio
Table 8 Geometric specifications of circular cutouts (the effect of cutout number increase)

\begin{tabular}{llll}
\hline $\begin{array}{l}\text { Cutout } \\
\text { number }\end{array}$ & $d$ cutout $(\mathrm{cm})$ & $h(\mathrm{~cm})$ & $\begin{array}{l}\text { Polar coordination } \\
\text { of cutout }(\mathrm{Rad})\end{array}$ \\
\hline 1 & 50,80 & $2 d$ & 0 \\
2 & 50,80 & $2 d, 4 d$ & $0, \frac{\pi}{2}$ \\
3 & 50,80 & $2 d, 4 d, 6 d$ & $0, \frac{\pi}{2}, \pi$ \\
4 & 50,80 & $2 d, 4 d, 6 d, 8 d$ & $0, \frac{\pi}{2}, \pi, \frac{3 \pi}{2}$ \\
\hline
\end{tabular}

of 500 and 1,000. The buckling capacity keeps reducing with increasing the cutout number in the width trend of the shell which will be more obvious in the cutouts of higher diameters.

Moreover, increase in the cutout number in the height trend has no significant effect on the buckling load reduction. This means that in case of cutout formation in the shells of $10 \mathrm{~m}$ diameter and diameter to thickness ratio of 1,000 , the buckling capacity is reduced about $30 \%$. The buckling capacity is reduced about $40 \%$ with increasing
Fig. 16 Displacement of cylindrical shell with circular cutout (shell diameters $20 \mathrm{~m}$, cutout diameter $100 \mathrm{~cm}$ ):

a $h=d, d=100 \mathrm{~cm}$,

$D=20 \mathrm{~m} ; \mathbf{b} h=2 d$,

$d=50 \mathrm{~cm}, D=15 \mathrm{~m}$;

c $h=4 d, d=50 \mathrm{~cm}$,

$D=20 \mathrm{~m}$

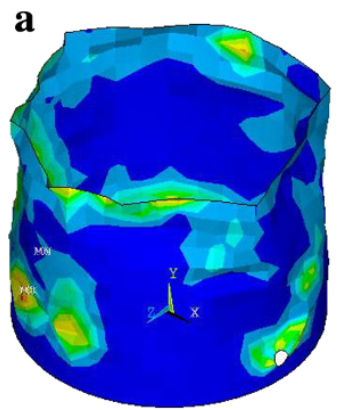

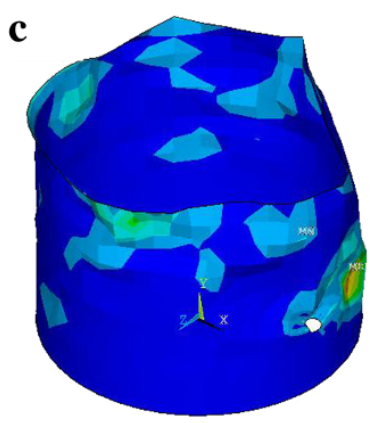




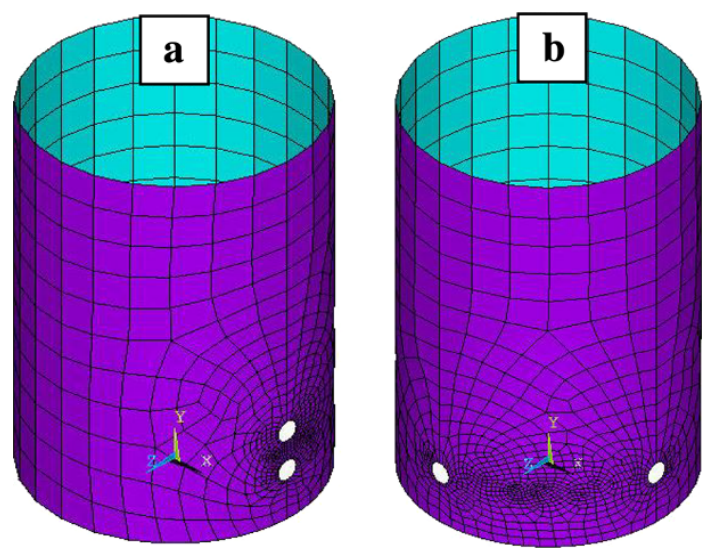

Fig. 17 Geometry and model of finite element of steel cylindrical shell with cutout: a increasing the cutout number in the shell height; b increasing the cutout number in the shell circumference

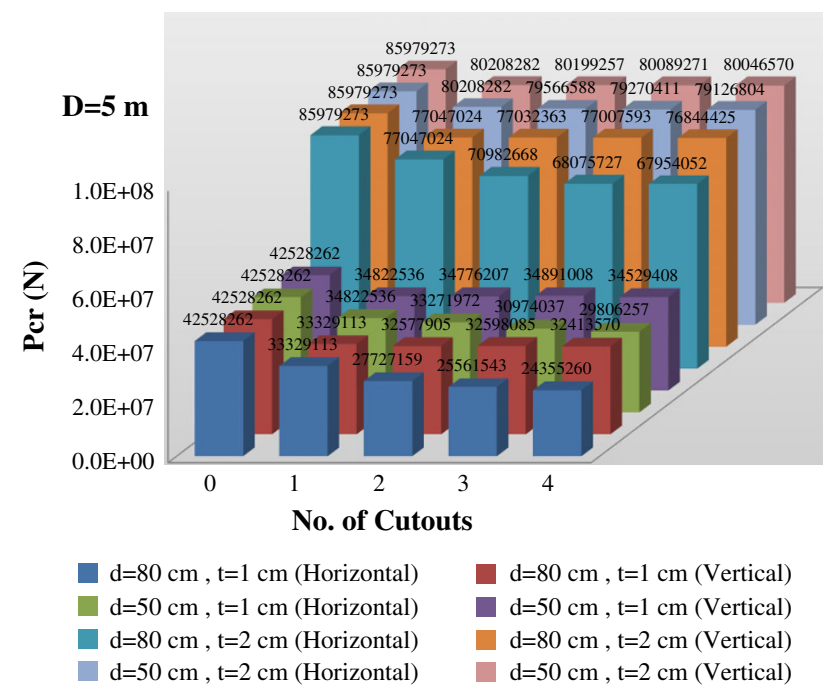

Fig. 18 The effect of number of cutouts on and their arrangement on buckling force of cylindrical shells (shell diameter $5 \mathrm{~m}$, shell thickness 10 and $20 \mathrm{~mm}$ ) the cutout number in the width strike in the presence of 50 and $80 \mathrm{~cm}$ cutouts. The buckling capacity reduction level is lower in case of cutout formation in the shells with $10 \mathrm{~m}$ diameter and diameter to thickness ratio of 500 as the wall is thicker compared to that of previous status. In average, the buckling capacity of the shell is reduced about 10-15\% (Fig. 21).

The buckling load is more reduced in case of increasing the diameter of cylindrical shell compared to the smaller diameter, assuming that the cutout diameter is constant. This reduction will be changed with almost equal slopes in both cases if the cutout number increases (Fig. 22). Figures 23 and 24 show the deformation of cylindrical shell, with increasing the cutout number in height and width strikes, under axial compression.

\section{Conclusion}

In this research, the steel cylindrical shells with circular cutouts have been subjected to axial compression and the results obtained through nonlinear numerical analysis are summarized as follows:

- The presence of cutout with any diameter decreases the load tolerance capacity of cylindrical shells about 10-35\%.

- Single cutout makes no significant change in the slope of force-displacement curve of the cylindrical shell in the elastic and post-buckling zones. In other words, single cutouts do not noticeably change the pre- and post-buckling behavior of cylindrical shells.

- Changing the cutout diameter from 300 to $1,000 \mathrm{~mm}$ has no significant effect on the local buckling capacity of the shell.

- Thinner shells are more susceptible to the cutouts compared to thicker ones. Cutouts reduce the local
Fig. 19 The percentage of buckling force reduction per cutout number $(D=5 \mathrm{~m}$, $t=10$ and $20 \mathrm{~mm})$

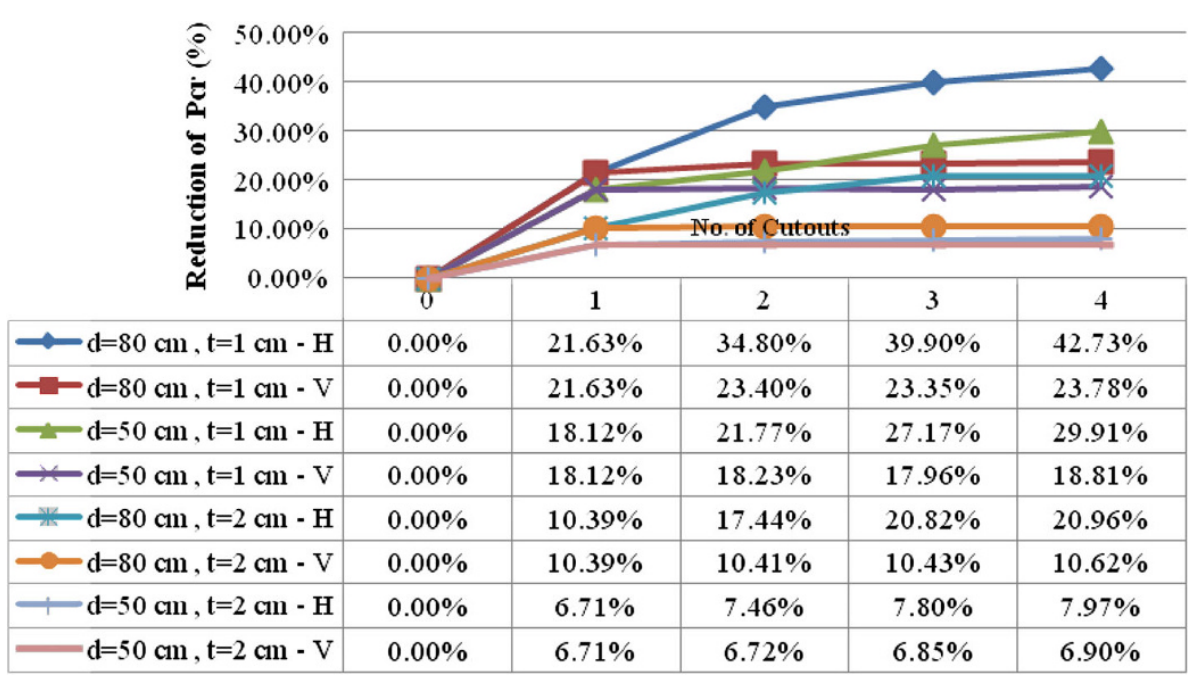




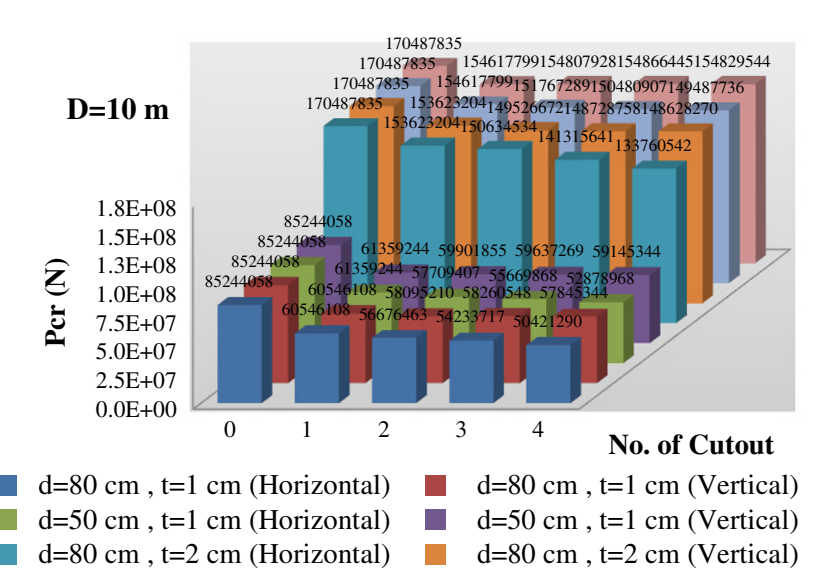

Fig. 20 Buckling force versus cutout number (shell diameter $10 \mathrm{~m}$, shell thickness 10 and $20 \mathrm{~mm}$ )

buckling capacity of shell about 10-15\% in the cylindrical shells, with the diameter to thickness ratio of less than 1,000. Meanwhile in shells with diameter to thickness more than 1,000 , such cutouts reduce the shell capacity about $30-35 \%$.

- Changing the elevation of cutout from cylindrical shell bottom has no significant effect on the shell buckling capacity and post-buckling behavior.

- The buckling capacity is reduced with increasing the cutout number in the shell width (horizontal arrangement of cutouts) and this reduction is more obvious in the cutouts with larger diameters. The shell buckling capacity is reduced about $30-45$ and $20 \%$ in the shells of $5 \mathrm{~m}$ diameter with the diameter to thickness ratio of 500 and 250 , respectively. In the shells of $10 \mathrm{~m}$ diameter and the diameter to thickness ratio of 1,000
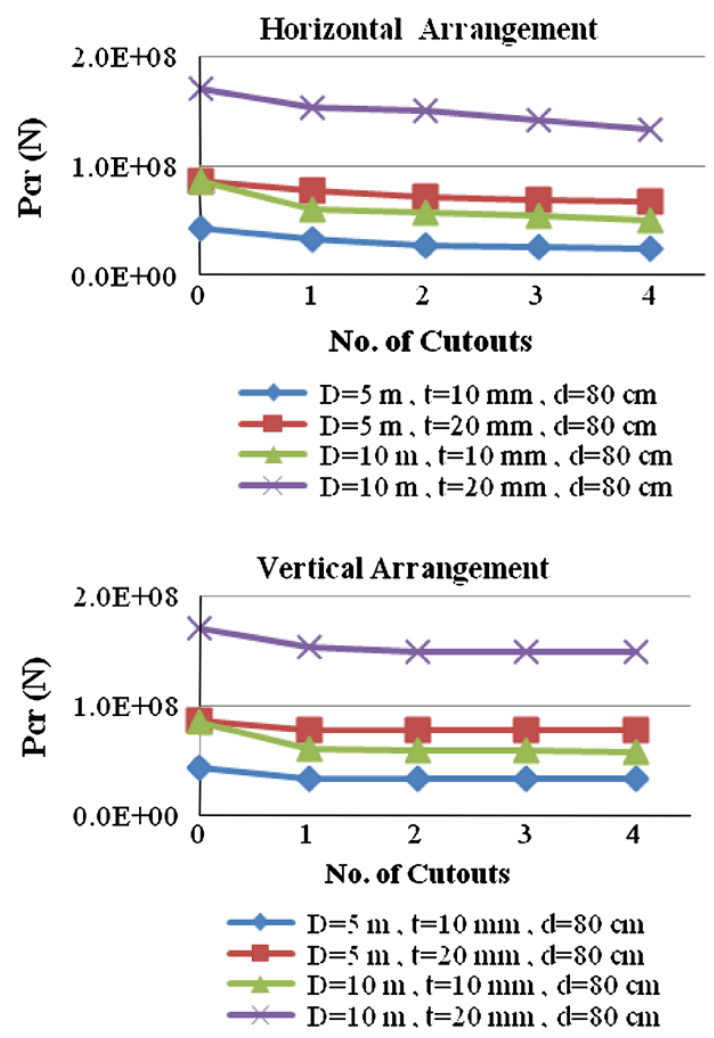

Fig. 22 Buckling force versus cutout number in shells of different $D /$ $t$ ratios

and 500, the shell buckling capacity is reduced about 40 and 10 to $15 \%$, respectively.

- The increasing cutout number in the shell height (vertical arrangement of cutouts) has no significant effect on the buckling load reduction.
Fig. 21 Reducing percentage of buckling force versus cutout number $(D=10 \mathrm{~m}, t=10$ and $20 \mathrm{~mm})$

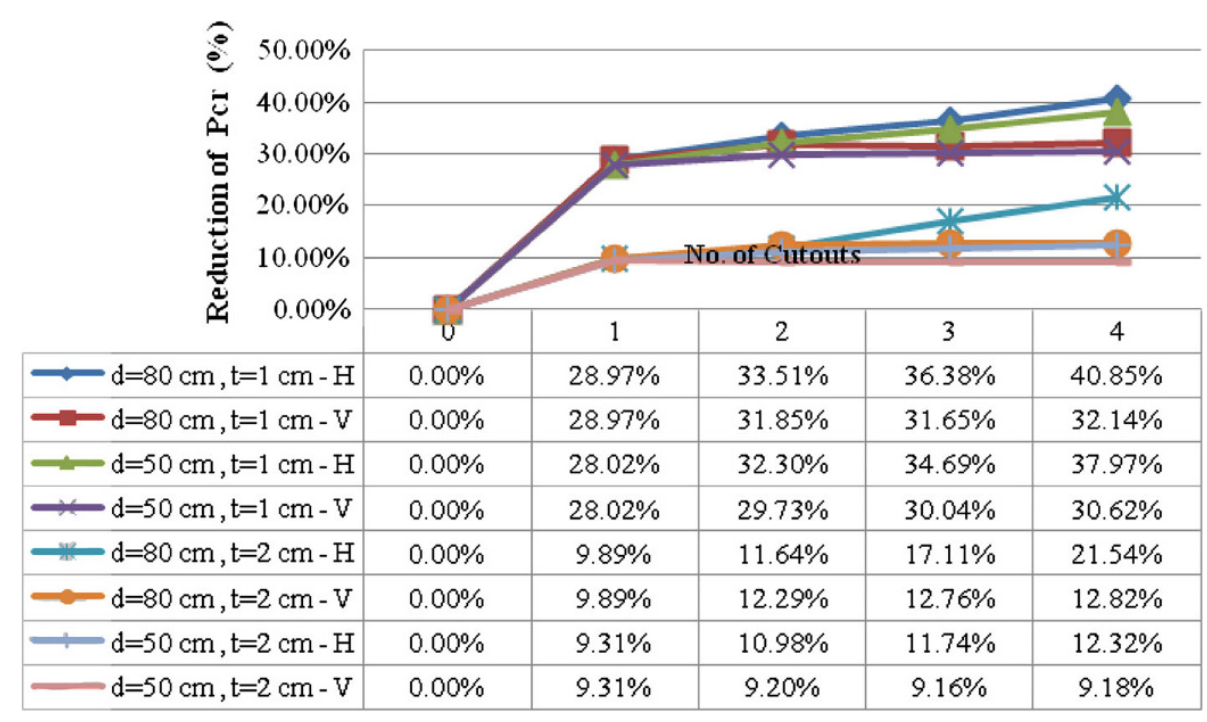



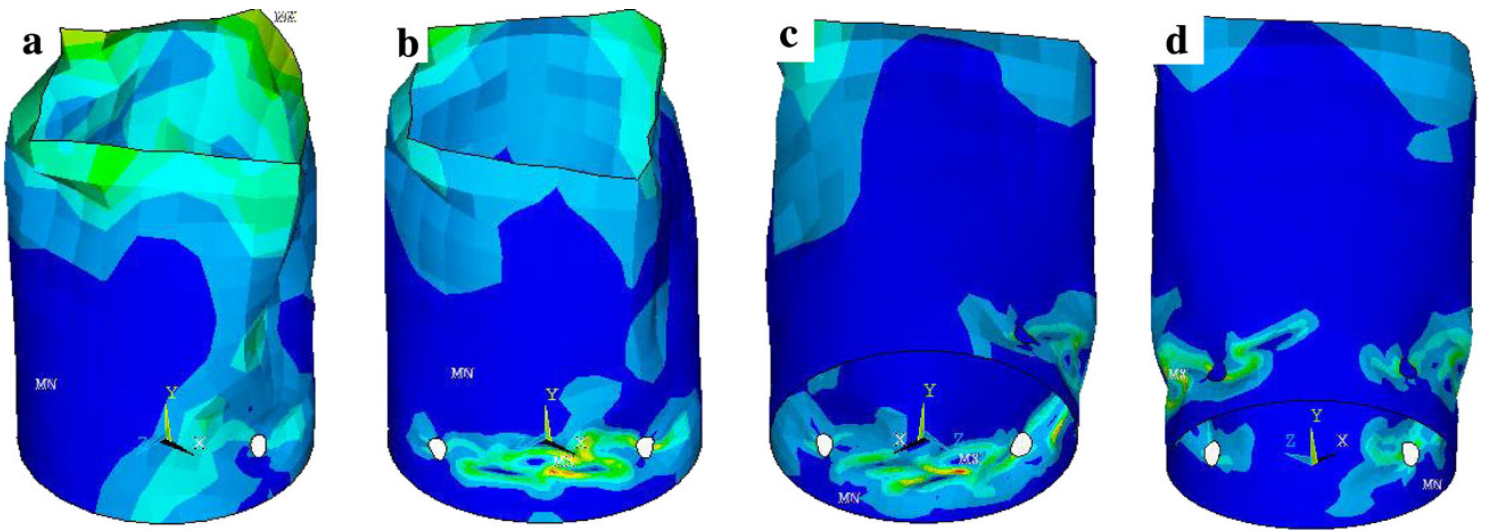

Fig. 23 Deformation of cylindrical shell with cutout (increasing the cutout in the width trend of shell): $\mathbf{a}=10 \mathrm{~m}, 1$ cutout; $\mathbf{b} D=10 \mathrm{~m}$, 2 cutouts; c $D=10 \mathrm{~m}, 3$ cutouts; $\mathbf{d} D=10 \mathrm{~m}, 4$ cutouts
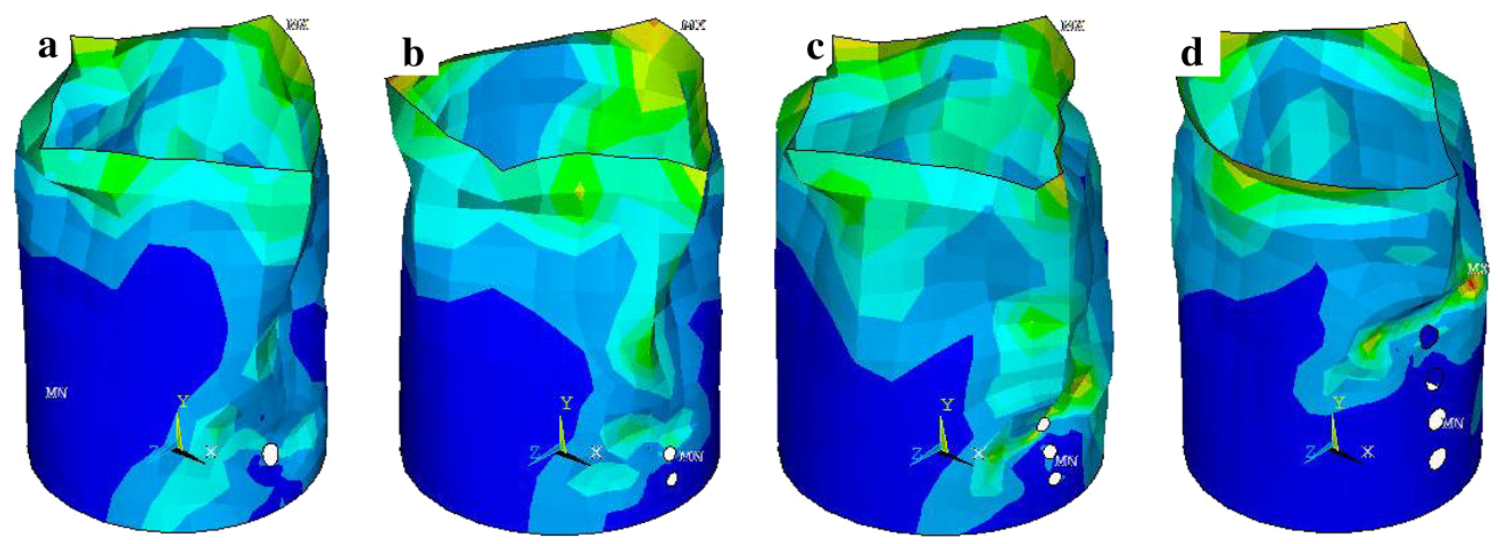

Fig. 24 Deformation of cylindrical shell with cutout (increasing the cutout in the height trend of shell): a $D=10 \mathrm{~m}, 1 \mathrm{cutout} ; \mathbf{b} D=10 \mathrm{~m}$, 2 cutouts; c $D=10 \mathrm{~m}, 3$ cutouts; d $D=10 \mathrm{~m}, 4$ cutouts

Open Access This article is distributed under the terms of the Creative Commons Attribution License which permits any use, distribution, and reproduction in any medium, provided the original author(s) and the source are credited.

\section{References}

ANSYS (2009) ANSYS users' manual. ANSYS Inc., USA

Arbocz J, Hol JMAM (1991) Collaps of axially compressed cylindrical shells with random amperfections. AIAA J 29:2247-2256

Brogan FA, Almorth BO (1970) Buckling of cylindrical shells with cutout. AIAA J. 8(2):236-240

Han H, Cheng J, Taheri F (2006) Numerical and experimental investigations of the response of aluminum cylinders with a cutout subject to axial compression. Thin-Walled Struct 44:254-270

Hilburger MW, Starnes J, Waas A (1998) The response of composite cylindrical shells with cutouts and subjected to internal pressure and axial compression loads. AIAA J-98-1768, A98-25060

Jullien JF, Limam A (1998) Effect of openings on the buckling of cylindrical shells subjected to axial compression. Thin Wall Struct. 31:187-202
Shariati M, Mahdizadeh Rokhi M (2010) Buckling of steel cylindrical shells with an elliptical cutout. Steel Struct 10(2):193-205

Starnes J (1970) The effect of a circular hole on the buckling of cylindrical shells. Ph.D. thesis, California Institute of Technology

Tafreshi A (2002) Buckling and postbuckling analysis of composite cylindrical shells with cutout subjected to internal pressure and axial compression load. Int $\mathbf{J}$ Pressure Vessel Piping 79:351-359

Tennyson RC (1968) The effects of unreinforced circular cutout on the buckling of circular cylindrical shells under axial compression. J Ebg For Industry ASME 90:541-546

Timoshenko SP, Gere JM (1961) Theory of elastic stability, 2nd edn. McGraw-Hill pub. Co., New York

Toda S (1983) Buckling of cylinders with cutouts under axial compression. Exp Mech 23(4):414-417

Van Dyke P (1965) Stresses about a circular hole in a cylindrical shells. AIAA J 33(9):1733-1742

Yeh MK, Lin MC, Wu WT (1999) Bending buckling of an elastoplastic cylindrical shell with a cutout. Eng Struct 21:996-1005 